\title{
Selective phonemic and semantic coding in short-term recall
}

\author{
EVELYN F. GOLDSTEIN \\ Cornell University, Ithaca, New York 14850
}

\begin{abstract}
Probed recall of targets from six-word sequences containing either phonemically similar, semantically similar, or control (i.e., unrelated phonemically and semantically) stimuli was compared under two conditions of encoding. For the semantic encoding condition, in which all cues provided category information about targets to be recalled, semantic similarity interference effects and phonemic similarity facilitation effects were demonstrated. In the phonemic encoding condition, in which all targets were cued by words which rhymed with them, only phonemic similarity interference effects were found.

These results were taken to provide further evidence contradictory to the coding-based dichotomization of primary and secondary memory, and to reflect the importance of selective coding strategies induced by task requirements as determinants of interference effects in short-term recall.
\end{abstract}

Although individual models of the postperceptual memory system may differ greatly in their detailed conceptualizations, they can be seen to fall into broad classes based on common general features. For example, duplexity models (e.g., Atkinson \& Shiffrin, 1968; Waugh \& Norman, 1965) postulate a dichotomization of the memory system into separate short-term and long-term stores. The extremely labile short-term store, referred to as primary memory (PM) in this paper, is the theoretical storage system assumed to be of principal importance in situations of short-term retention. Such situations typically involve the single presentation of a small amount of material followed by a recall or recognition test within a few seconds. On the other hand, secondary memory (SM), the relatively stable long-term store, is assumed to be functioning in long-term retention, typified by situations involving multiple presentations of a large amount of material tested after much longer delays.

One of the major arguments used in support of a memory dichotomy stems from research indicating that short-term memory and long-term memory tasks are differentially affected by phonemic (i.e., articulatory/acoustic) and semantic similarity and therefore reflect different types of coding. Initially, short-term retention was found to involve coding along a phonemic, as opposed to semantic, dimension. For long-term retention the reverse was found to be the case (see Shulman, 1971, for a review of the relevant literature). More recent research, however, demonstrating the occurrence of semantic processing in some short-term memory tasks (e.g., Calfee \& Peterson,

This research is based on a dissertation submitted to cornell University in partial fulfillment of the requirements for the Ph.D. degree. The author wishes to thank her supervisor, $U$. Neisser, for his generous and thoughtful counsel. Thanks are also due to $H$. Bernbach and $G$. Wilcox for their aid in designing the model for data analysis and their many helpful comments. Joel Norman's helpful criticiam of an earlier draft of this paper is also greatly appreciated.

Requests for reprints should be sent to Evely n Goldstein, now at the Department of Psychology, University of Winnipeg, Winnipes, Manitoba, Canada.
1968; Wickens, 1970) and the occurrence of phonemic coding in long-term retention (e.g., Buschke \& Lenon, 1969; Dale \& McGlaughlin, 1971) has led to a revision of the coding-based dichotomy formulation. It is now proposed that while SM can involve both semantic and phonemic coding, PM involves phonemic coding exclusively (e.g., Baddeley, 1970; Baddeley \& Patterson, 1971). Moreover, certain short-term memory paradigms are taken to involve not only PM, but SM to some extent. Thus Baddeley (1972) has attributed some recent evidence for semantic coding in short-term retention as due to the underlying contribution of SM rather than PM in the tasks concerned.

Techniques have been developed (see Watkins, 1974, for a review and comparison of these methods) which attempt to separate the PM and SM components in several short-term memory tasks. Studies based on these techniques have not been conclusive in their support of the coding-based memory dichotomy. Whereas some studies have found the estimated PM component to be sensitive to phonemic factors only (e.g., Kintsch \& Buschke, 1969), others have found PM to be unaffected by phonemic factors (e.g., Glanzer, Koppenaal, \& Nelson, 1972; Watkins, Watkins, \& Crowder, 1974) or affected by semantic, as opposed to phonemic similarity (e.g., Craik \& Levy, 1970).

Additional research which indicates that the adoption of a two-store model based on coding differences is unwarranted has been examined by Shulman (1971). His review suggests that when subjects are free to control their own coding strategy, semantic coding is not used as freely as phonemic coding in PM. Given task demands that require it, however, semantic coding is found to be fully as salient in PM as phonemic coding.

The importance of task demands as a variable in determining the subject's processing strategies has recently been recognized (e.g., Butterfield, Belmont, \& Peltzman, 1971; Frost, 1972; Tversky, 1973). Several studies clearly demonstrate that subjects can vary the 
type of attribute coding strategy they employ as a result of instructions or task demands. Tversky (1969) has shown that stimulus coding in a short-term recognition task occurs along either a visual or verbal dimension as a function of the subjects' expectations regarding the mode of presentation of the recognition test material. Selective semantic coding in a short-term probe recognition task as a function of task demands has been demonstrated by Shulman (1972). Buschke and Lenon (1969) have shown subjects to code both phonemic and semantic stimulus attributes for long-term word recognition. However, when the task requirements provided them with prior knowledge as to which information was relevant for performance, they coded selectively along either the phonemic or semantic dimension, whichever was appropriate. Cermak, Schnorr, Buschke, and Atkinson (1970) demonstrated that subjects instructed to weight differentially the attention paid to processing the phonemic or semantic features of stimulus words presented in a long-term recognition task, were able to attend-selectively, although not exclusively, to particular word qualities. Klein and Klein (1974) have demonstrated similar selective phonemic and semantic coding as a function of task conditions in a short-term recognition task.

The studies which have been instrumental in demonstrating selective attribute coding as a function of task demands have all employed recognition memory paradigms. The present study was designed to demonstrate the existence of selective coding of phonemic $v$. semantic information in a short-term probed recall task. The paradigm employed was modeled after that of Bregman (1968). On each trial, a six-word stimulus sequence containing phonemically similar, semantically similar, or control (i.e., unrelated on these dimensions) material was presented. The subjects were subsequently probed for retrieval of a target word in the sequence on the basis of qualifying cues containing either phonemic or semantic information about the target.

This paradigm involves what is considered to be PM storage. One of the techniques for separating PM and SM components in short-term retention tasks views responses as being retrieved from PM if no more than a critical number of other items (either further stimulus items or responses) intervenes between an item's presentation and its subsequent recall. Tulving and Colotla (1970) have selected seven items as the critical number, whereas Craik (1970) has chosen six. On all trials in the following experiment, less than the critical number of six items intervened between a target's presentation and subsequent test. Thus, retrieval from $\mathrm{PM}$, as defined by the above criterion, was being tapped.

It should be noted that the exact degree of intrasequence similarity for phonemically similar and semantically similar stimulus material cannot be determined and equated. However, it is assumed that ther exist sufficient essential parallels between the processes involved in cued recall of targets from sequences containug phonemically similar tems and the processes involved in cued recall of targets from sequences containing semantically similar items to warrant a comparison of these operations. In recall of targets from both types of sequences, interference is assumed to arise due to the fact that items within the secuences share common features (i.e., ei:her common phonemic or common semantic features), and recall of target words is made difficult as a rtsult of the processing of these common features (cf. Smith, Shoben, \& Rips, 1974).

It was predicted that under task conditions stressing optimal semantic coding, semantic similarity interference effects would be demonstrated. That is, it was predicted that under such conditions recall of targets from sequences containing semantically similar items would be decreased relative to recall of targets from control sequences, in which the items were neither semantically nor phonemically similar. Thus the following research bears directly upon the question of whether or not semantic coding occurs in PM. Furthermore, it was predicted that if subjects can selectively code along either the phonemic or semantic dimension, then under conditions stressing maximal phonemic coding and minimizing semantic coding, these semantic similarity interference effects would no longer be evident. Conversely, it was predicted that under conditions stressing optimal semantic coding, the phonemic similarity interference effects commonly found in short-term retention would be eliminated. That is, recall of targets from phonemically similar sequences was expected not to differ significantly from recall of targets from control sequences under conditions stressing optimal semantic, as opposed to phonemic, coding.

\section{METHOD}

\section{Subjects}

Forty eight undergraduate male students at Cornell University served as volunteer subjects.

\section{Stimulus Materials}

Three paper booklets were prepared for each subject. Each booklet was composed of a series of 12 six-word sequences and their subsequent cued recall tests. The start of each sequence was marked by a blank yellow page. Each stimulus word was typed in capital letters in the center of a separate white page.

Three types of sequence appeared in each booklet:

Phonemically similar (P) sequences. Within a $P$ sequence the words sounded alike, containing the same vowel sounds and/or having consonants in common; e.g., NIECE, LEAF, FEET, NEED, FIELD, FEAST.

Semantically similar (S) sequences. Within an $S$ sequence the words were all exemplars of some obvious superordinate category; e.g., HORSE, WOLF, LION, BEAR, SKUNK, TIGER.

Control (C) sequences. Within a $C$ sequence words were unrelated as to phonemic quality and category membership; e.g., VEST, HIL L, JOKE, PERFUME, BLOOD, SWAN. 
An attempt was made to equate the word stimuli across the three sequence types with regard to number of syllables, frequency, and the number of words with ambiguous lexical classification. Nine six-item sequences of each ty pe (P, S, and C) were constructed for use in the main part of the experiment. Eleven additional $C$ sequences were constructed, nine of which served as buffer sequences (i.e., recall of items from these sequences was recorded but not scored). The remaining two $\mathrm{C}$ sequences were used in an auxiliary part of the experiment.

In each sequence one of the stimulus words served as the recall target (i.e., to-be-recalled item). A recall cue appeared on a white page following the last word of each sequence. There were two types of recall cue:

Phonemic recall cue. A word which rhymed with the target; e.g., Target word - "VEST"; Recall cue - "____ thymes with PRESSED." All rhyming words were chosen to be as graphically dissimilar as possible from the target words, in order to minimize the contribution of visual or formal similarity components.

Semantic recall cue. The name of a taxonomic category to which the target belonged; e.g., Target word - "VEST"; Recall cue -“" is an ARTICLE OF CLOTHING."

A suitable phonemic and semantic recall cue were constructed for each target. In the case of $P$ sequences, with stimuli similar in sound, each target was chosen such that it did not rhyme with any other words in the sequence. Thus the phonemic recall cue rhymed only with the target and could serve to access it alone; e.g., in the P sequence example given above, the target "NIECE" was tested with the phonemic cue " LEASE," and the semantic cue " rhymes with the case of $S$ sequences, the targets were chosen such that, in addition to belonging to the overall superordinate category of the sequence, they also could be further classified under a different category name (usually a category subordinate to the main, superordinate one). This alternate category name was used as the semantic cue to access the target. In the $S$ sequence example presented above, the target "HORSE" was tested with the semantic cue "___ is a FARM ANIMAL" and the phonemic cue "—_ rhymes with COURSE."

A postexperimental questionnaire asking for short definitions of all target words was administered to all experimental subjects in order to ensure that the phonemic and semantic cues used in the research were relevant for each subject individually.

\section{Procedure}

A between-subjects design was employed to test retention under two conditions of encoding: (1)Phonemic Encoding (PE)Subjects were presented with phonemic cues after all stimulus sequences. It was hypothesized that this manipulation would lead subjects to place a greater emphasis on processing the phonemic properties of the stimuli. (2)Semantic Encoding (SE) Subjects were tested with semantic cues only. It was hypothesized that in this condition greater emphasis would be placed on processing the semantic features of the stimuli. Twelve subjects, tested individually, served in each of these two conditions. Assignment of subjects to conditions was random by order of appearance in the laboratory. Subjects in the two conditions received identical instructions except that examples of recall cues were appropriate for the given condition. Subjects were not given prior information as to the structure of the sequences. They were told there was no penalty for guessing when they could not remember the target word in a sequence.

Tone signals instructing subjects to turn the pages in a booklet were presented over a tape recorder at $3-\mathrm{sec}$ intervals. Thus the stimulus duration and recall interval, as well as the intersequence interval, were all slightly less than $3 \mathrm{sec}$ (i.e., $3 \mathrm{sec}$ minus the time taken to turn a page). The subject's oral response to the recall cue at the end of each sequence was recorded only if it occurred during the allotted recall interval.

Subjects worked through three consecutive booklets. Additional blank pages at the end of each booklet prevented subjects from knowing when a booklet would end. Three-minute rest intervals occurred between booklets, during which the instructions were briefly repeated.

All target words appeared in one of three locations: the first, third, or fifth serial position of a sequence. Thus the cued recall test occurred at the end of a retention interval (RI) of 6,12 , or $18 \mathrm{sec}$.

The first three sequences in all booklets formed a set of buffer $C$ sequences. These sequences were included in order to familiarize the subjects with the procedure and to reduce general primacy effects. The order of presentation of the three sequences in the initial buffer set in each booklet was fixed across subjects. All three RIs $(6,12,18 \mathrm{sec})$ were tested in each buffer set, and each RI was tested equally often in the first, second, and third sequence over booklets. The data from these buffer sequences were not scored.

In the remaining nine sequences of a booklet, each of the three sequence types $(P, S$, and $C)$ was tested once at each of the three RIs $(6,12$, and $18 \mathrm{sec})$. Thus each booklet comprised one complete replication of the experimental conditions. Across subjects within each encoding condition ( $P E$ and $S E$ ), all sequences were tested equally often at all three RIs, and equally often in the first, second, and third booklets. In addition, no more than two sequences of the same sequence type occurred successively in a booklet, nor were more than two successive sequences tested at the same RI

The subjects were matched in pairs across the two conditions such that the two members of a pair received a set of booklets identical in every way except for the recall cue pages, which were appropriate to the encoding condition. For each matched pair of subjects, the five nontarget words in each sequence were distributed randomly in the serial positions remaining after the target had been fixed in the appropriate serial position (determined by the RI to be tested for the sequence).

At the end of the third booklet in both conditions, two auxiliary $C$ sequences were tested as an integral part of the booklet. The order of these two $\mathrm{C}$ sequences was counterbalanced across subjects, and in both sequences the targets were tested at an 18-sec RL. In the PE condition, the first of these sequences, $P E\left(C_{2}\right)$, was tested with a phonemic cue, whereas the second sequence, PE $\left(C_{2}\right)$, was tested with a "surprise" semantic cue. Similarly, in the SE condition, the first sequence, $S E\left(C_{1}\right)$, was tested with a semantic cue, while a "surprise" phonemic cue followed the second sequence, $\mathrm{SE}\left(\mathrm{C}_{2}\right)$.

\section{Control Procedures}

The relatively fast presentation rate and short recall interval used in this experiment were, in part, an attempt to minimize guessing effects. In addition, the target items were chosen such that both the phonemic and semantic cues for each item elicited the target item as a first guess with low frequency in a pretested free guessing situation.

As the major control for guessing effects, two control groups, the phonemic encoding control (PEC) group and the semantic encoding control (SEC) group, each consisting of 12 subjects, were tested. These 24 control subjects were randomly assigned to groups by order of appearance in the laboratory and were tested individually.

In the control groups, subjects in each condition were matched to subjects in the corresponding experimental groups, such that the same 12 sets of booklets were used. However, in the control groups, the target items were removed from all experimental sequences. The remaining stimuli and recall cues were not altered in any way. The sequence orderings in each set of booklets remained as in the experimental conditions, with the following modification. From the first three sequences (i.e., the buffer $\mathrm{C}$ set) an item other than the target was chosen at random for removal. For each matched pair of control subjects, these three $\mathrm{C}$ sequences, still containing targets, were distributed randomly among the other sequences in a booklet. Thus, three 
Table 1

Mean Proportion Correct Target Recall for All Treatment Conditions

\begin{tabular}{lccccc}
\hline $\begin{array}{l}\text { Encoding } \\
\text { Condition }\end{array}$ & $\begin{array}{c}\text { Sequence } \\
\text { Type }\end{array}$ & \multicolumn{5}{c}{ Retention Interval } & \\
& $6 \mathrm{Sec}$ & $12 \mathrm{Sec}$ & $18 \mathrm{Sec}$ & \\
\hline Phonemic & $\mathrm{P}$ & .389 & .333 & .528 & $(.056)$ \\
Encoding & $\mathrm{S}$ & .778 & .750 & .750 & $(.176)$ \\
& $\mathrm{C}$ & .583 & .556 & .639 & $(.028)$ \\
Semantic & $\mathrm{P}$ & .833 & .722 & .833 & $(.000)$ \\
Encoding & $\mathrm{S}$ & .556 & .611 & .667 & $(.093)$ \\
& $\mathrm{C}$ & .472 & .806 & .778 & $(.000)$ \\
\hline
\end{tabular}

Note-Numbers in parentheses indicate guessing probabilities for $P, S$, and $C$ sequence targets in the corresponding control groups, PEC and SEC.

of the twelve five-item sequences in a control booklet contained targets, whereas the nine experimental sequences contained no targets. The targets in the two auxiliary $\mathrm{C}$ sequences in the third booklet were also removed.

Subjects in both control groups received instructions similar to those for the experimental groups, except that they were warned that a sequence might or might not contain a target. They were asked to provide best guesses to recall cues in the event that there was no target. In PEC, all cues provided were phonemic, except for the "surprise" semantic cue occurring after the final sequence. Similarly, all cues in SEC were semantic, except for the final "surprise" phonemic cue.

The guessing probabilities as assessed from the data of these control groups were used to correct the data of experimental subjects for the effects of pure contextual guessing.

\section{RESULTS}

The raw data for the major analysis were the 27 responses of each experimental subject, three responses for each sequence type $(P, S, C)$ by $R I(6,12,18 \mathrm{sec})$ condition. (The data from the final two $C$ sequences in Booklet 3 for each subject were analyzed separately and did not enter into the major analysis.) For each subject, the proportion of items correct, $P(C)$, at each Sequence Type by RI was scored. Mean $P(C)$ s for $P, S$, and $C$ sequence targets at each $\mathrm{RI}$ in the $\mathrm{PE}$ and $\mathrm{SE}$ conditions are presented in Table 1.

Subjects' $P(C)$ scores were then corrected for guessing using the guessing data from the PEC and SEC groups. The proportion of correct guesses, $P(G)$, was calculated separately for each type of sequence in both control groups. The $P(G)$ was equal to $x / 108$, where $x$ is the observed number of correct guesses for nine sequences by twelve control subjects. The resulting $P(G)$ s for $P, S$, and $C$ sequences in PEC and SEC are presented in Table 1.

If the standard correction for guessing formula were to be applied to the data, then the proportion scores of subjects in a given experimental condition, PE or SE, for target recall in a particular sequence type tested at each RI would be corrected for guessing by solving for P(T) in the following formula:

$$
P(C)=P(T)+[1-P(T)] \times P(G),
$$

where $\mathrm{P}(\mathrm{C})$ is a subject's observed proportion correct for the given sequence type tested at a given $R I, P(T)$ is the true probability of knowing the item, and $P(G)$ is the guessing probability for the appropriate sequence type and cue, as estimated from the data of the appropriate control group. However, in cases where $P(C)$ equals zero or one, and $P(G)$ does not equal zero, the formula is inapplicable, for it yields $P(T)$ which remains unchanged [if $P(C)=1$ ] or is negative [if $P(C)=0$ ]. Since such cases occurred in the data, an alternate correction for guessing procedure was adopted, and a subsequent transformation applied to the data to convert the corrected scores to an index $\left(\mathrm{d}^{\prime}{ }_{\mathrm{A}}\right)$ similar in interpretation to the traditional performance measure, $\mathrm{d}^{\prime}$ (Green \& Swets, 1966).

The signal detection measure, $d^{\prime}$, has been previously employed successfully in measuring recall performance (Bernbach, 1967). It has a distinct advantage over the standard correction for guessing formula in that it does not make the implicit assumption that retention is an all-or-none process but allows for the retention of partial information about the to-be-remembered items (Kintsch, 1970).

The correction for guessing employed was based upon the assumption that the information for making a correct response provided by a recall cue alone is supplemented by information in the memory trace of a target item, when the latter is available. When cues, but not targets, are presented (i.e., in the control groups) it is assumed that there exists a distribution of cue informativeness, $\mathrm{I}_{Q}$, (i.e., information about the target provided by the sequence context and recall cue) across subjects and recall cues, for a particular sequence type. When subjects are presented with the target item (i.e., in the experimental conditions), added information, $I_{M}$, (i.e., information about the target provided by the memory trace of the item) becomes available for target identification. In the control tasks only $I_{Q}$ is available, whereas in the experimental tasks both $I_{Q}$ and $I_{M}$ are available, resulting in the distribution of informativeness, $I_{Q}+I_{M}$. It is further assumed that subjects will make a correct response when the information for a target item exceeds a given criterion. These assumptions imply that there exists a hypothetical performance curve of $P(C) v$. $P(G)$ as this criterion is varied. (This performance curve is similar in interpretation to the ROC curve associated with signal detection theory, in which $\mathrm{I}_{\mathrm{Q}}$ is equivalent to $\mathrm{N}$, the noise distribution, and $\mathrm{I}_{Q}+\mathrm{I}_{\mathrm{M}}$ is equal to $\mathrm{SN}$, the signal plus noise distribution.)

Even though the shapes of the distributions of $I_{Q}$ and $I_{Q}+I_{M}$ are unspecified in this model, one may obtain criterion-free indices of performance. The performance index adopted in the present experiment, $\mathrm{d}_{\mathrm{A}}^{\prime}$; (cf. Simpson \& Fitter, 1973) was based on an estimate of the area (A) under the performance curve. The procedure employed was similar to that recommended by Pollack, Norman, and Galanter (1964) for a nonparametric 
assessment of performance in recognition memory experiments. The value of $\mathrm{A}$ was estimated on the basis of a two-line approximation to the performance curve, where the lines intersect at the observed data point $[\mathbf{P}(\mathrm{G}), \mathrm{P}(\mathrm{C})]$; so that

$$
A=[1+P(C) \cdot P(G)] / 2 .
$$

Next, the index $d_{A}^{\prime}$ used for data analysis was computed by a method (described in Green \& Swets, 1966, pp. 217-219) for converting area estimates (A) to corresponding values of $d^{\prime}$ (Note 1 ). It is presumed that the sampling distribution of the statistic $\mathrm{d}_{\mathrm{A}}^{\prime}$ estimated in this way more closely approximates a normal distribution than does the sampling distribution of $A$ itself (Simpson \& Fitter, 1973).

The mean $d_{A}^{\prime} \dot{s}$ resulting for each Sequence Type by RI combination for the two experimental conditions are shown in Table 2.

A three-way analysis of variance (Encoding Condition by Sequence Type by RI) was performed on the $d_{A}^{\prime}$ scores. The main effect of encoding condition was significant, $[F(1 / 22)=14.96, p<.001]$, as were the effects of sequence type, $[F(2 / 44)=4.30, p<.05]$, and the interaction of Encoding Condition by Sequence Type $[F(2 / 44)=35.95, p<.001]$.

Analysis of key simple main effects showed that the effect of encoding condition was not significant for $S$ sequences $[F(1,66)<1]$, but was significant for both $P$ sequences $[F(1 / 66)=60.62, p<.001]$ and $C$ sequences $[F(1 / 66)=4.93, p<.05]$. The effect of sequence type was significant in both $\mathrm{PE}[\mathrm{F}(2 / 44)=10.81, \mathrm{p}<.001]$ and $\mathrm{SE}[\mathrm{F}(2 / 44)=29.41, \mathrm{p}<.001]$ conditions.

Since neither the main effect of RI nor higher order interactions involving RI proved to be significant, the data were pooled across this factor for multiple comparisons of sequence type effects in the PE and SE conditions. The mean $\mathrm{d}^{\prime}{ }_{\mathrm{A}} \mathrm{s}$ collapsed over RIs are presented in Table 2. Multiple $t$ tests were performed, and the critical values of $t$ used in tests of significance were computed by the "method of adjusted significance levels" recommended by Ryan (1960). In PE, mean $d_{A}^{\prime}$ for $P$ sequences was significantly lower than mean $d_{A}^{\prime}$ for both $S$ sequences [t $(44)=4.08, p<.01]$ and $C$ sequences $[t(44)=3.94, p<.01]$. The means for $C$ and $S$ sequences did not differ significantly $[t(44)=0.14]$. In $S E$, mean $d_{A}^{\prime}$ for $P$ sequences was significantly greater than mean $\mathrm{d}_{\mathrm{A}}^{\prime}$ for both $\mathrm{C}$ sequences $[\mathrm{t}$ $(44)=3.00, p<.01]$ and $S$ sequences $[t(44)=7.63$, $\mathrm{p}<.01]$ Mean $\mathrm{d}_{\mathrm{A}}^{\prime}$ for $\mathrm{S}$ sequences was significantly lower than that for $C$ sequences $[t(44)=3.73, p<.01]$.

Data from the final two $\mathrm{C}$ sequences presented to the subjects in Booklet 3 were subjected to a separate analysis. For each sequence, the subjects received a score of zero (incorrect or no response) or one (correct response). The overall proportion correct for each sequence was as follows: $\operatorname{PE}\left(C_{1}\right)=0.75, \operatorname{PE}\left(C_{2}\right)=0.08$,
$\mathrm{SE}\left(\mathrm{C}_{1}\right)=0.75, \mathrm{SE}\left(\mathrm{C}_{2}\right)=0.58$. Since $\mathrm{P}(\mathrm{G})$ as assessed from the data of subjects in the control conditions was zero for each of these sequences, the proportions correct remain unchanged when corrected for guessing in the standard correction for guessing procedure outlined earlier. In each encoding condition, $\mathrm{PE}$ and $\mathrm{SE}$, a Cochran Q test for dichotomous data was conducted on the subjects' scores on the final two $\mathrm{C}$ sequences. Performance on $\operatorname{PE}\left(C_{1}\right)$ was significantly superior to performance on $\mathrm{PE}\left(\mathrm{C}_{2}\right), \quad(\mathrm{Q}=8.00, \mathrm{p}<.005)$. Performance on $\operatorname{SE}\left(C_{1}\right)$ and $\operatorname{SE}\left(C_{2}\right)$ did not differ significantly, $(\mathrm{Q}=0.67, \mathrm{NS})$.

\section{DISCUSSION}

The results of the present study demonstrate that different interference effects can arise in short-term probed recall as a function of different coding strategies induced by task requirements. For the PE condition, results were as predicted. When subjects knew in advance that targets were always to be tested with phonemic cues, they presumably adopted an encoding strategy which maximized the attention paid to processing the phonemic attributes of the stimulus material and minimized attention to the semantic attributes. Under such conditions, targets in phonemically similar (P) sequences were less well recalled than targets in control (C) sequences, whose items were phonemically unrelated. Moreover, as a result of minimal coding along the semantic dimension in this condition, the semantic similarity of $S$ sequence stimuli had no interfering effect upon recall; targets in $S$ sequences and in $C$ sequences were recalled equally well. This result obtained since $C$ and $\mathrm{S}$ sequences contained items which were equally unrelated with regard to phonemic properties, and maximum attention had been paid to coding these phonemic attributes in the PE condition.

The presumption that subjects in PE were selectively processing phonemic, as opposed to semantic, information is further substantiated by the comparison of recall for $\operatorname{PE}\left(C_{1}\right)$ and $\operatorname{PE}\left(C_{2}\right)$ sequence targets. When subjects received a "surprise" semantic cue, their recall of the target was significantly worse than recall of a target in an equivalent sequence tested with a phonemic cue, and was so poor, in fact, that the overall proportion of correct recall was nearly zero. Thus it appears that PE

Table 2

Mean $d_{A}^{\prime}$ for All Treatment Conditions

\begin{tabular}{lcrrrr}
\hline Encoding & Sequence & \multicolumn{4}{c}{ Retention Interval } \\
Condition & Type & 6 Sec & 12 Sec & 18 Sec & Mean \\
\hline Phonemic & $\mathrm{P}$ & .719 & .585 & 1.051 & .785 \\
Encoding & $\mathrm{S}$ & 1.390 & 1.363 & 1.363 & 1.372 \\
& $\mathrm{C}$ & 1.270 & 1.190 & 1.595 & 1.352 \\
& $\mathrm{P}$ & 2.510 & 2.072 & 2.510 & 2.364 \\
Semantic & $\mathrm{S}$ & 1.061 & 1.208 & 1.525 & 1.265 \\
Encoding & $\mathrm{C}$ & .916 & 2.471 & 2.019 & 1.802 \\
\hline
\end{tabular}


subjects encoded little or no semantic information about the stimulus material.

In the SE condition, designed to maximize semantic coding and minimize phonemic coding, the results were only in part as predicted. Targets in S sequences were indeed less well recalled than targets in $C$ sequences, demonstrating that semantic coding can occur in PM. Moreover, under conditions stressing maximal semantic coding, phonemic similarity interference effects did not occur. Recall for $\mathrm{P}$ sequence targets, however, exceeded that for $\mathrm{C}$ sequence targets, indicating the existence of a phonemic similarity facilitation effect. If $\mathrm{SE}$ subjects had, in fact, been attending only minimally to the phonemic properties of the stimulus material and had been encoding primarily along the semantic dimension, then equivalent recall for $P$ and $C$ sequence targets should have resulted, for there is no reason to believe a priori that items within $P$ sequences should be less interfering on the basis of their semantic properties than items within $C$ sequences.

The hypothesis that phonemic information about sequence stimuli was being registered in addition to semantic information in the $S E$ condition receives further support from the comparison of recall performance on targets in sequences $\operatorname{SE}\left(C_{1}\right)$ and $\operatorname{SE}\left(C_{2}\right)$. If $\mathrm{SE}$ subjects were encoding both phonemic and semantic information, then it seems plausible that at the time of the "surprise" phonemic test they could scan back and retrieve the target on the basis of its phonemic qualities, if it were still available in memory. The SE subjects were able to retrieve the target on the "surprise" phonemic test considerably well [overall proportion correct recall of $\left.S E\left(C_{2}\right)=0.58\right]$, and performance on $\operatorname{SE}\left(C_{2}\right)$ was not significantly poorer than performance on $\operatorname{SE}\left(C_{1}\right)$, tested with an accustomed semantic cue.

Phonemic information about the stimuli may have been registered in memory in the SE condition by virtue of the fact that the subjects were unable to reduce phonemic coding to a minimum, or chose not to do so because they found it beneficial for the recall of $\mathbf{P}$ sequence targets. The phonemic facilitation effect demonstrated in SE can easily be explained if such was the case. Despite possible interference along the phonemic dimension in $P$ sequences, phonemic coding in $S E$ could aid $P$ sequence target recall in the following way. On those trials in which a target from a $P$ sequence could not be recalled in $S E$, subjects could have supplemented the semantic information given in a cue (e.g., "-_-_- is a RELATTVE") with the phonemic information available as to the general sound of the $P$ sequence (e.g., "all items contained an / / / phoneme") to restrict guessing (in the case of the example provided, to a set of words which were kinship terms, and, in addition, contained an /e/ phoneme; arriving at the target word "NIECE"). For C sequences, however, stimuli had separate and distinct phonemic qualities, so phonemic information about sequence items wouid have been of no aid in restricting guesses.

Although the data from the control group SEC were used to correct the data of subjects in SE, only pure guessing effects were eliminated. The guessing correction procedure would not completely correct for such "restricted set" guessing, because subjects in SEC did not know that the targets in $P$ sequences which they were attempting to guess were similar phonemically to other items in the sequence. The subjects in SE, having seen the targets, were aware of this constraint. An examination of the guesses of SEC subjects as to missing targets in $\mathrm{P}$ sequences supported this hypothesis. Only 3 of the possible 108 guesses were remotely similar in sound to either the missing $P$ sequence targets or other words in the sequences; all other guesses were only semantically related to the cues.

It should be noted parenthetically that a comparable hypothesis regarding "restricted set" guessing could be applied to $\mathrm{S}$ sequences in the $\mathrm{PE}$ condition. The $\mathrm{PE}$ subjects, when tested with a phonemic cue at the end of an $S$ sequence for which the target could not be recalled, should have been able to restrict guessing to items which rhymed with the cue word and were members of the superordinate category of the sequence. Such restricted guessing in PE would result in semantic facilitation effects for $\mathrm{S}$ sequence target recall relative to $\mathrm{C}$ sequence target recall, where restricted guessing was not possible. The fact that a significant semantic facilitation effect was not found lends further support to the conclusion that virtually no semantic information was being registered in the PE condition.

Inspection of the error data in PE and SE indicated that the exact nature of the interference arising as a result of processing the common features of items in $P$ sequences and $S$ sequences may have been different. The results suggest that under phonemic coding in PE, the processing of phonemically similar items in $P$ sequences may have sometimes led to the loss of adequate discriminability among item traces. Phonemic cues used to probe $P$ sequence targets in $P E$ sometimes incorrectly accessed either words other than the target within the sequence, or words which were erroneous conglomerates of some of the phonemes present in the P sequence (i.e., "smudged" traces). These erroneous conglomerates in fact rhymed with the target if only the initial phoneme of the target was "smudged." On the other hand, under semantic coding in $S E$, errors in $S$ sequences usually resulted from the subjects' failure to respond, suggesting that interference may have resulted from the fact that semantic coding of $S$ sequence items in this condition did not always proceed to the deeper subordinate level, rendering the cues unable to access any target item.

As a rough check of this hypothesis, each subject's errors in each sequence type were classified as either omissions (no response given) or intrusions (a response other than the target given). For each subject, the 
proportion of intrusion errors (I) out of total errors for a given sequence type was determined. In each encoding condition the mean I for each seauence type was calculated. The largest mean I was found for $P$ sequences in $\mathrm{PE}(\overline{\mathrm{I}}=0.21)$. The intrusion errors for $\mathrm{P}$ sequences in $\mathrm{PE}$ were in all cases either intrasequence intrusions or "conglomerate trace" intrusions. Moreover, mean I for $P$ sequences in $P E$ was significantly greater $[t(22)=1.72$, one-tailed $p<.05]$ than mean I for $S$ sequences in $\mathrm{SE}$ $(i=0.08)$. Thus the suggested hypothesis receives some support. In contrast to the above, the mean Is for $\mathrm{C}$ sequences under $\mathrm{PE}(\overline{\mathrm{I}}=0.16)$ and $\mathrm{C}$ sequences under $\mathrm{SE}$ $(\mathrm{I}=0.18)$ did not differ significantly, [correlated $\mathrm{t}$ $(11)=0.18, \mathrm{NS}]$.

The lack of a significant effect of retention interval in this experiment is somewhat surprising. The data showed neither a bow-shaped serial position curve over RIs nor a normal forgetting curve, with decreasing performance as a function of test delay. These results of no differential forgetting over the retention interval of 6 to $18 \mathrm{sec}$ in both PE and SE are in contrast to those of Bregman (1968), who, in a similar task, found forgetting to increase rapidly over this retention interval when recall was tested with either phonemic or semantic cues. His design, however, employed a very long sequence of stimuli presented for retention, with recall tests interspersed within the sequence, as opposed to the short, independent sequences used here. There is some evidence (Johnston, Griffith, \& Wagstaff, 1972) that, when semantic category cues are used as recall probes for items in short lists, serial position curves are significantly flatter than those obtained for recall tested by positional probes, and are eliminated altogether for semantic cue performance when reaction time measures are used.

The phonemic and semantic cues used in this experiment do not appear to be equal in their efficacy of probing targets in $\mathrm{C}$ sequences. Recall of $\mathrm{C}$ sequence targets was significantly higher when semantic cues were used. This result is in keeping with that of Bregman (1968), who found recall for target words imbedded in a long sequence of phonemically and semantically unrelated words to be best when tested with semantic cues. Moreover, Nelson, Wheeler, Borden, and Brooks (1974) have found that under conditions stressing semantic processing of list words, subsequent semantic synonym cues are more effective than subsequent phonemic rhyme cues as retrieval aids in a cued recall task. The rather surprising result in the present study that recall for $S$ sequence targets in $P E$ was not significantly greater than recall for $\mathrm{S}$ sequence targets in SE can be explained in light of these findings. This lack of difference may be attributed to the fact that $S$ sequence target recall in SE was somewhat inflated by having been probed with the more effective type of cue.

In summary, the results of the present study clearly indicate that whether or not item similarity will lead to interference effects depends on the coding strategies adopted by subjects. These coding strategies appear to be largely determined by specific task demands. On the one hand, semantic coding appears to be an optional coding strategy which subjects can employ in short-term retention if it is found to be advantageous or if the task demands it, but which can be eliminated if it is not required. On the other hand, the results suggest that phonemic coding is of a more basic nature; perhaps fundamentally linked with the process of stimulus acquisition itself. The findings reported here are best incorporated within a theoretical framework which views memory as a continuum, and considers phonemic and semantic coding to reflect different levels or depths of processing (Cermak, 1972; Craik \& Lockhart, 1972; Nelson et al., 1974). Moreover, much of the phonemic v. semantic coding literature appears to be easily interpreted in terms of this "levels of processing" approach (cf. Cermak, 1972; Craik \& Lockhart, 1972). It appears that the issue of whether or not the memory system should be divided into theoretically distinct short- and long-term stores would be more appropriately decided on the basis of evidence other than coding differences (Wickelgren, 1973). Perhaps a more fruitful approach to the study of acquisitional processes and coding operations would be to disregard the dichotomous framework of PM and SM and focus on the investigation of differences in selection and retention of attributes as a function of the processing demands imposed by specific stimulus material, experimental paradigms, and task requirements.

\section{REFERENCES}

Atkinson, R. S., \& Shiffin, R. M. Human memory: A proposed sy stem and its control processes. In $K$. W. Spence \& J. T. Spence (Eds.), The psychology of learning and motivation. Vol. 2. New York: Academic Press, 1968.

Baddeley, A. D. Effects of acoustic and semantic similarity on short-term paired-associate learning. British Journal of Psy chology, 1970, 61, 335-343.

Baddeley, A, D. Retrieval rules and semantic coding in short-term . memory. Psychological Bulletin, 1972, 78, 379-385.

Baddeley, A. D., \& Patterson, K. Relation between long-term and short-term memory. British Medical Bulletin, 1971, 27, 237-242.

Bernbach, H. A. Decision processes in memory. Psychological Review, 1967, 74, 462-480.

Bregman, A. S. Forgetting curves with semantic, phonetic, graphic, and contiguity cues. Journal of Experimental Psychology, 1968, 78, 539-546.

Buschke, H., \& Lenon, R. Encoding homophones and sy nonyms for verbal discrimination and recognition. Psychonomic Science, 1969, 14, 269-270.

Butterfield, E. C., Belmont, J. M., \& Peltzman, D. J. Effects of recall requirement on acquisition strategy. Journal of Experimental Psychology, 1971, 90, 347-348.

Calfee, R. C., \& Peterson, R. E. Effect of list organization on short-term probe recall. Journal of Experimental Psychology, 1968, 78, 468-474.

Cermak, G., Schnorr, J., Buschke, $H, \&$ Atkinson, $R, C$. Recognition memory as influenced by differential attention to semantic and acoustic properties of words. Psychonomic Science, 1970, 19,79-81.

Cermak, L. S. Human memory. New York: Ronald Press, 1972. Craik, F. I. M. The fate of primary memory items in free recall. Journal of Verbal Learning and Verbal Behavior, 1970, 9, 143-148. 
Craik, F. I. M., \& Levy, B. A. Semantic and acoustic information in primary memory. Journal of Experimental Psychology, $1970,86,77-82$.

Craik, F. I. M.. \& Lockhart, R. S. Levels of processing: A framework for memory research. Journal of Verbal Leaming and Verbal Behavior, 1972, 11,671-684.

Dale, H. C. A., \& McGlaughlin, A. Evidence of acoustic coding in long-term memory. Quarterly Journal of Experimental Psychology, 1971, 23, 1-7.

Frost. N. Encoding and retrieval in visual memory tasks. Journal of Experimental Psychology, 1972, 95, 317-326.

Glanzer, M., Koppenaal, L., \& Nelson, R. Effects of relations between words on short-term storage and long-term storage. Journal of Verbal Learning and Verbal Behavior, 1972, 11, 403-416.

Green, D. M., \& Swets, J. A. Signal detection theory and psychophysics. New York: Wiley, 1966.

Johnston, W. A., Griffith, D., \& Wagstaff, K, R. Speed, accuracy and ease of recall. Joumal of Verbal Learning and Verbal Behavior, 1972, 11, 512-520.

Kintsch, W. Models for free recall and recognition. In D. A Norman (Ed.), Models of human memory. New York: Academic Press, 1970.

Kintsch, W. \& Buschke, H. Homophones and synonyms in short-term memory. Joumal of Experimental Psychology. $1969,80,403.407$.

Klein, G. A.. \& Klein, H. A. The influence of serial retention and theme identification paradigms on encoding. Quarterly Journal of Experimental Psy chology, 1974, 26, 556-560.

Nelson, D. L.. Wheeler, J. W., Borden, R. C., \& Brooks, D, H. Levels of processing and cueing: Sensory versus meaning features. Journal of Experimental Psychology, 1974, 103, 971-977.

Pollack, I., Norman, D. A., G Galanter, E. An efficient non-parametric analysis of recognition memory. Psy chonomic Science, 1964, 1, 327-328.

Ryan, T. A. Multiple comparisons in psychological research. Psychological Bulletin, 1959, 56, 26-47.

Shulman, H, G. Similarity effects in short-term memory. Psychological Bulletin, 1971, 75, 399-415.

Shulman, $H$. $G$. Semantic confusion errors in short-term memory. Joumal of Verbal Leaming and Verbal Behavior. 1972, 11, 221-227.
Simpson, A. J., \& Fitter, M. J. What is the best index of detectability? Psychological Bulletin, 1973, 80, 481-488.

Smith, E. E., Shoben, E. J., Rips, L. J. Structure and process in semantic memory: A feature model for semantic decisions. Psychological Review, 1974, 81, 214-241.

Tulving, E., \& Colotla, V. A. Free recall of trilingual lists. Cognitive Psychology, 1970, 1, 86-98.

Tversky, B. Pictorial and verbal encoding in a short-term memory task. Perception \& Psychophysics, 1969, 6, 225-233. Tversky, B. Encoding processes in recognition and recall. Cognitive Psy chology, 1973, 5, 275-287.

Watkins, M. J. Concept and measurement of primary memory. Psychological Bulletin, 1974,81, 695-711.

Watkins, M. J., Watkins, O. C., \& Crowder, R. G. The modality effect in free and serial recall as a function of phonological similarity. Journal of Verbal Learning and Verbal Behavior. $1974,13,430-447$.

Waugh, N. C.. \& Norman, D. A. Primary memory. Psychological Review, 1965, 72, 89-104.

Wickelgren, W, A. The long and short of memory. Psychological Bulletin, 1973, 80, 425-438.

Wickens, D. D. Encoding categories of words: An empirical approach to meaning. Psychological Review, 1970, 77, 1-15.

\section{NOTE}

1. For each sequence type in the PEC and SEC conditions, $P(G)$ was the ratio $x / 108$, where $x$ is the observed number of correct guesses out of 9 sequences times 12 subjects. If $x=0$ then the formula for $A$ results in an infinite value for $d_{A}$ when $P(C)=1$. Therefore $P(G)$ was assigned the value $1 / 109$ when $x$ was equal to zero. This procedure results in a value of $d^{\prime} A$ which is larger than any other observed value of $d^{\prime} A$ for the same value of $P(C)$. Since the specific value assigned to $P(G)$ in the event that $x$ was zero had to be arbitrarily chosen, the obtained values of $\mathrm{d}^{\prime} \mathrm{A}$ could not be equivalent to those of the traditional index, of

(Received for publication March 28, 1975. Accepted April 11, 1975.) 